\title{
Non-linear Distortion Noise Cancellation for Satellite Return Links
}

\author{
Svilen Dimitrov \\ German Aerospace Center (DLR), Satellite Networks Department, 82234 Wessling, Germany, e-mail: svilen.dimitrov@dlr.de
}

\begin{abstract}
In this paper, an iterative receiver that performs non-linear distortion noise cancellation is presented. The performance is assessed for time division multiple access (TDMA), orthogonal frequency division multiple access (OFDMA), and single-carrier frequency division multiple access (SC-FDMA) waveforms for application over the satellite return link channel. The performance is assessed in terms of energy efficiency and spectral efficiency, where the total degradation (TD) of the received signal-to-noise ratio (SNR) is minimized. The modulation formats of the DVB-RCS2 satellite return link standard and a respective non-linear channel have been used. Two iterations of non-linear noise cancellation across the different modulation orders have shown the following total degradation gains: $1.1-2.5$ dB in OFDMA, $0.3-1.1$ dB in SC-FDMA, $0.1-0.8$ dB in TDMA with $20 \%$ roll-off, and $0.2-1.9$ dB in TDMA with $5 \%$ roll-off.

Index Terms-Non-linear noise cancellation, satellite communications, TDMA, OFDMA, SC-FDMA, spectral efficiency.
\end{abstract}

\section{INTRODUCTION}

Due to the ongoing shift towards more bandwidth demanding applications and services, next generation satellite networks need to offer both higher throughput and higher data rates, flexibility to adapt to traffic demand across the coverage area, and at the same time decrease the cost per transmitted bit. The utilization of larger pieces of bandwidth in the higher radio frequency (RF) bands, such as Ka, Q and $\mathrm{V}$ bands, imposes hardware implementation challenges, and imperfections cause signal distortion. Therefore, suitable signal processing techniques at the transmitter and/or receiver that maximize information rate of the link are required.

High-throughput satellite [1] communications suffer from non-linear distortion that originates from the non-linear transfer characteristics of the high-power amplifiers onboard the satellite and at the user terminal. These adverse effects reduce the power and spectral efficiencies of the transmission waveform. Time division multiple access (TDMA) is employed in the return (RTN) satellite link according to the DVB-RCS2 standard [2]. Waveforms such as orthogonal frequency division multiple access (OFDMA) and single-carrier frequency division multiple access (SC-FDMA) are at the heart of terrestrial mobile long-term evolution (LTE) networks due to their high spectral efficiency (SE) and flexible resource allocation. However, these waveforms have been recently considered for application over satellite for the sake of vertical network hand-over [1]. The performance of SC-FDMA and OFDMA has been compared in the satellite return link with amplifier characteristics in the $\mathrm{K}$ and $\mathrm{S}$ bands in [3]. In this study, a novel receiver is developed that performs iterative cancellation of the intermodulation interference (IMI) from the non-linear distortion, and it is tested for practical amplifier characteristics in $\mathrm{Ka}$ band in TDMA, OFDMA, and SC-FDMA waveforms over the satellite return link channel.

In state-of-the-art systems, the IMI is reduced by means of power control, such as output back-off (OBO) [3, 4]. However, this approach is known to penalize the energy efficiency, when high OBO is applied. In addition, a number of pre-distortion techniques can be applied at the transmitter, such as signal pre-distortion [5], static and dynamic data pre-distortion [6] and waveform pre-distortion [7]. Cancellation of the IMI has been first proposed in [8] for OFDM systems. The approach has been adopted for systems with SC-FDMA in [9]. The interfering component is estimated by means of a model for the received constellation centroids based on the Bussgang decomposition [10], where the cross-correlation between the transmitted and received symbols is exploited, and a single cross-correlation factor is used for all the symbols in the constellation. This approach is suitable for systems, where the non-linear distortion noise is uncorrelated with the symbols, such as in OFDMA. However, when the IMI is correlated with the signal, such as in SC-FDMA and TDMA, individual scaling factors for each constellation point are required.

In this paper, a symbol-based equalizer with non-linear noise cancellation is proposed for application at the gateway in the satellite return link. The performance of the improved receiver is compared with static data pre-distortion at the transmitter [6] which is used in state-of-the art satellite systems. The improved receiver enables operation with minimum total degradation (TD) at a much lower optimum $\mathrm{OBO}$ for all the three considered TDMA, OFDMA, and SC-FDMA waveforms. Two, or even one, iterations of non-linear noise cancellation are sufficient to provide significant energy efficiency gains.

The rest of the paper is organized as follows. Section II describes the satellite transmission chain in the return link. Section III presents the non-linear distortion effects and the iterative receiver for IMI cancellation. Section IV elaborates on the performance evaluation results. Finally, Section V concludes the paper.

\section{SATELlite TRANSMISSION CHAIN IN RETURN LINK}

In the satellite return link, a stream of forward error correction (FEC) encoded bit packets are mapped to a stream of symbols from a given constellation at the modulator. The modulation formats of the DVB-RCS2 satellite return link standard [2] include quadrature phase shift keying (QPSK), 8-level phase shift keying (8-PSK) and 16-level quadrature 


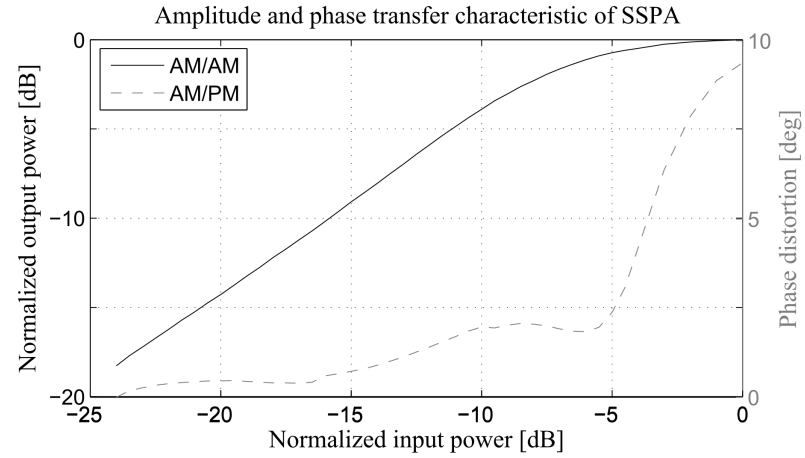

Fig. 1: AM/AM and AM/PM characteristics of a Ka-band SSPA at the user terminal.

amplitude modulation (16-QAM). Time division multiple access (TDMA) is applied in the state-of-the-art satellite return link. In this study, also OFDMA and SC-FDMA waveforms are considered. The waveform selection and processing is represented as a type of symbol multiplexing. In TDMA, the information symbols are pulse shaped by means of a square root raised cosine filter (SRRCF) to ensure spectrum integrity for RF transmission. After digital-to-analog conversion (DAC) and up-conversion to the carrier frequency, e.g., in Ka band, the signal is amplified by means of a solid state power amplifier (SSPA) in single-carrier mode at the user terminal. Practical input amplitude/output amplitude (AM/AM) and input amplitude/output phase (AM/PM) characteristics of a Ka-band SSPA are presented in Fig. 1.

The satellite transponder in the return link is assumed to be operated in multi-carrier mode with, e.g., more than 40 carriers [1]. Therefore, the channel in the satellite link can be considered as flat-fading over the user bandwidth. In this multi-carrier setup, the memory and non-linear distortion effects of the satellite transponder are transformed into Gaussian noise which directly reduces the link budget. As a result, the SSPA operated in single-carrier mode at the user terminal can be considered the main contributor to the non-linear distortion.

At the gateway receiver, the signal is amplified by means of a low-noise amplifier (LNA), and it is distorted by additive white Gaussian noise (AWGN). After down-conversion to baseband and analog-to-digital conversion (ADC), a matched SRRCF is applied. The symbols are then demultiplexed according to the underlying waveform, e.g., TDMA, OFDMA, or SC-FDMA. Finally, the demodulator and the decoder perform are applied to obtain the received packets.

The digital baseband signal at the input of the demodulator at the gateway receiver can be expressed in TDMA as follows:

$$
\mathbf{y}=\mathbf{h}_{\mathrm{SRRCF}} *\left(h F\left(\mathbf{h}_{\mathrm{SRRCF}} * \mathbf{x}\right)\right)+\check{\mathbf{w}},
$$

where $\mathbf{x}$ is the transmitted symbol vector, $\mathbf{h}_{\mathrm{SRRCF}}$ is the impulse response of the SRRCF filter, $*$ is the linear convolution operator, $F(\cdot)$ is the non-linear transfer function of the SSPA, $|h|^{2}$ is the channel gain, and $\check{\mathbf{w}}$ is filtered version of the the AWGN vector $\mathbf{w}$ after the SRRCF.

In state-of-the-art systems, non-linear distortion is com- monly compensated by pre-distortion at the transmitter. Signal pre-distortion is used to linearize the amplifier characteristic. It generally involves additional analog electronics [5], which implement the inverse of the amplifier characteristic. Signal pre-distortion is known to introduce unwanted out-of-band radiation. Data pre-distortion, on the other hand, is a purely digital technique [6], which preserves the signal spectrum. Here, the transmitted constellation is modified such that the received centroids are close to the original transmitted constellation. The first type of data pre-distortion, called static data pre-distortion, accounts for the static nonlinearities in the channel. The second type, called dynamic data pre-distortion, also compensates memory effects. It takes into account the current symbol, as well as the $(L-1) / 2$ symbols preceding and succeeding symbols, where $L$ is the memory depth. An alternative solution is band-limited waveform pre-distortion [7] of the effect of the non-linear device on the in-band portion of the pulse-shaped digital signal. Dynamic data pre-distortion and waveform pre-distortion are suitable solutions for application over the forward link satellite channel to counter nonlinear and memory effects. Since the main contributor to the non-linear distortion in the return link is the memoryless SSPA at the user terminal, the channel is also memoryless, and therefore static data pre-distortion is considered sufficient.

\section{ITERATIVE CANCELLATION OF NON-LINEAR DISTORTION}

In this Section, the effects of non-linear distortion on the received signal are discussed, and an iterative IMI cancelling receiver is presented.

\section{A. Non-linear distortion effects}

The non-linear distortion in the satellite channel introduces constellation warping and symbol clustering at the input of the demodulator at the receiver [4]. The IMI is dependent on the signal waveform, and therefore it contains useful information of the transmitted signal. In single-carrier systems, such as in TDMA, the interfering component is correlated with the transmitted symbols, and each constellation point at the receiver is individually warped, scaled, and rotated with respect to the symbol constellation at the modulator [4]. In OFDMA systems, where the analog carrier is digitally subdivided in a large number of subcarriers, as well as in multi-carrier systems with a large number of analog carriers, such as in the multi-carrier satellite transponder in the return link with TDMA, the large degree of symbol multiplexing results in a signal with a close to Gaussian distribution. According to the Bussgang theorem [10], the interfering component is in this case uncorrelated with the signal component, and it is transformed into Gaussian noise.

As the interfering component is dependent on the signal, it contains useful information. State-of-the-art non-linear compensation techniques do not attempt to extract the useful information from the interference component, and it is treated as noise to be minimized. The proposed symbol-based equalizer with non-linear noise cancellation employs low-complexity 


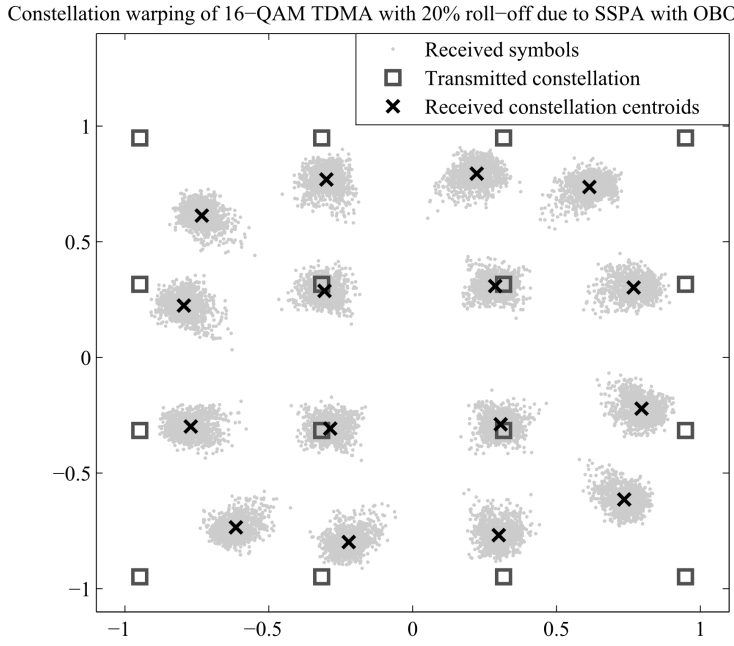

Fig. 2: Constellation warping of 16-QAM TDMA with $5 \%$ roll-off after an SSPA in the satellite return link.

cancellation of the interfering component at the receiver which results in a significant increase of the energy efficiency.

The following decomposition of the signal and the interfering component is employed, where the symbol at the output of the non-linear device is given as:

$$
F(x)=K x+d .
$$

The output signal, $F(x)$, is an attenuated and possibly rotated replica of the information-carrying symbol, $x$, plus non-linear distortion noise, $d$. The scaling factor $K$ is obtained as the covariance of the transmitted and received symbols normalized by the average transmitted symbol power. There is no general assumption on the distribution of the non-linear distortion noise, and it can be correlated or not with the signal.

The constellation warping effects of the non-linear distortion after the SSPA from Fig. 1 operated in single-carrier mode at the user terminal are presented in Fig. 2 for the received 16-QAM TDMA symbols with $20 \%$ roll-off and an OBO of $2 \mathrm{~dB}$. Next, the non-linear distortion effects on TDMA with $5 \%$ and $20 \%$ roll-off factors are compared with the effects on SC-FDMA and OFDMA. In TDMA, a frame of 2048 symbols is assigned to 32 users with 64 localized symbols per user. In SC-FDMA, as well as in OFDMA, the analog carrier is subdivided in digital subcarriers, whereby a portion of the subcarriers are dedicated to the user, and the rest are set to zero. Here, 2048 subcarriers are assigned to 32 users with 64 localized subcarriers per user. In Figs. 3 and 4, the amplitude of the attenuation factors and the variance of the non-linear distortion noise are presented as a function of the OBO. TDMA and SC-FDMA experience an almost identical warping of the amplitude of the received centroids. The three distinct symbol amplitudes in the 16-QAM constellation have three distinct attenuation factors and noise variances. The degree of attenuation and the noise variance correspond to the PAPR of the schemes. TDMA with $20 \%$ roll-off experiences the least distortion, followed by TDMA with $5 \%$ roll-off, SCFDMA, and OFDMA. It can be noticed that received symbols

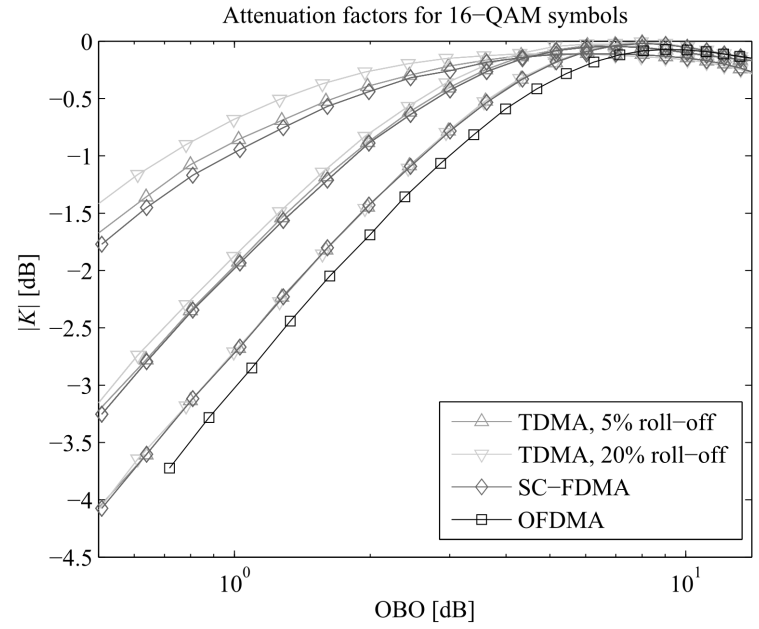

Fig. 3: Attenuation factor of the non-linear distortion for 16-QAM after an SSPA in the satellite return link.

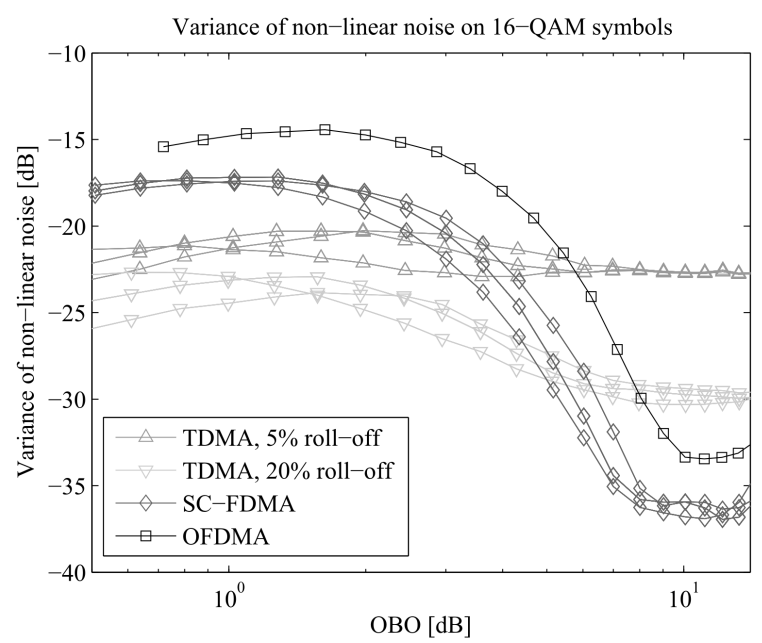

Fig. 4: Variance of non-linear distortion noise for 16-QAM with after an SSPA in the satellite return link.

in OFDMA are expectedly scaled by a single attenuation factor, and have the largest noise variance. It should be noted that the truncation of the SRRCF impulse response (16 periods are considered with oversampling factor of 8) to reduce the processing complexity and latency in TDMA contributes additional non-linear distortion noise which is evident in the high OBO region, and it is more prominent in TDMA with $5 \%$ roll-off.

\section{B. Iterative receiver for IMI cancellation}

The receiver can extract the useful signal information in the IMI by means of interference cancellation. It has been first proposed in [8] for systems with OFDM. A set of received symbols, e.g., a received frame, is buffered. In the first iteration, the symbols are demodulated in a maximum likelihood (ML) fashion. Based on the knowledge of the distortion function in the transmission chain, the demodulated symbols are used to obtain an estimate of the received symbols. In order to obtain an estimate of the interfering component, the demodulated symbols are scaled and subtracted from the 


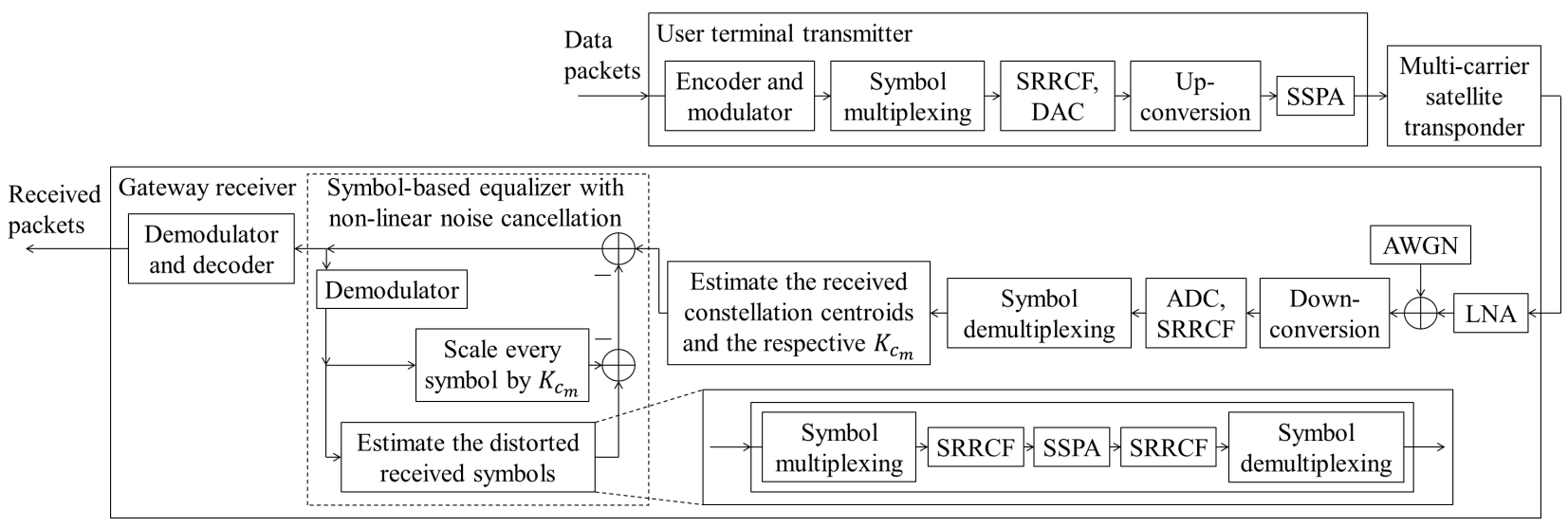

Fig. 5: Block diagram of the satellite transmission cain in the return link with an iterative IMI cancelling receiver.

estimate of the received symbols. In the original approach, one factor is used to scale all the constellation symbols. It is then subtracted from the buffered symbols to obtain an improved set of received symbols as an output of the first iteration of IMI cancellation. In the second iteration, the newly obtained received symbols are demodulated and used to better estimate the interfering component which is again subtracted from the originally buffered received symbols. The output of this process are the improved received symbols after a number of iterations. The IMI cancellation approach has been adopted for systems with SC-FDMA in [9]. However, the same estimator for the interfering component based on a single scaling factor for all symbols is applied. This approach is suboptimum for SC-FDMA and TDMA. Here, individual scaling factors for each constellation point need to be applied. In general, the number of distinct scaling factors corresponds to the number of distinct symbol amplitudes in the applied constellation, as shown in Fig. 3, due to the amplitude-dependent amplifier characteristics from Fig. 1.

The block diagram of the proposed improved receiver is presented in Fig. 5. After symbol demultiplexing, the received symbols are obtained and buffered for further processing. At this point, estimates of the received constellation centroids and the respective scaling factors, $K_{c_{m}}$, are calculated for every constellation point, $c_{m}, m=1, \ldots, M$, where $M$ is the modulation order. The scaling factors $K_{c_{m}}$ represent the ratio of a received centroid and the corresponding transmitted constellation point. In order to estimate the received constellation centroids, dedicated pilots have been introduced in the DVBS2X standard as part of the signalling for channel estimation. These received constellation centroids are also used as a reference for the demodulator and in the calculation of the log-likelihood ratios at the decoder.

In the first branch, the demodulated symbols are scaled by the respective scaling factors, $K_{c_{m}}$. In the second branch, an estimate of the distorted received symbols is computed, using the knowledge of the processing blocks and distortion function in the satellite chain, such as symbol multiplexing, SRRCF, SSPA, SRRCF and symbol demultiplexing. The scaled symbols are subtracted from the estimate of the received symbols to obtain an estimate of the interfering component, which is subtracted from the originally buffered received symbols to produce improved soft values for the received symbols after the first iteration. It is shown in Section IV that even one or two iterations can yield a significant gain.

\section{Performance COMParison}

The performance of the proposed receiver is evaluated in a Monte Carlo simulation in the satellite return link setup for three different transmission schemes, i.e., TDMA, SCFDMA and OFDMA. A large number of processed symbols are simulated, in order to obtain sufficient convergence of the statistics, e.g., up to 1000 error events per simulation point. A similar setup of the symbol frame as the one described in Section III is used. In TDMA, the $5 \%$ and $20 \%$ roll-off factors increase the bandwidth occupation of a carrier accordingly. It is shown in [11] that two contiguous aggregated analog carriers based on OFDMA or SC-FDMA can be employed with as little as $5 \%$ guard band. The improved IMI cancelling receiver is tested against the standard one from $[8,9,12]$ and against static data pre-distortion at the transmitter.

In order to quantify the energy efficiency penalty induced by the satellite channel, the TD metric is defined as follows:

$$
\mathrm{TD}[\mathrm{dB}]=\mathrm{OBO}+\mathrm{SNR} \underset{\substack{\text { required in } \\ \text { non-lin.chan. }}}{\text { SNR }}-\mathrm{SNR}_{\substack{\text { required in } \\ \text { lin.chan. }}} .
$$

In addition to the $\mathrm{OBO}$ penalty, the $\mathrm{TD}$ incorporates the penalty on the SNR requirement to achieve a target bit-error rate (BER) in the non-linear channel with respect to the SNR requirement in the linear channel with AWGN.

The results for the TD in QPSK and 16-QAM are presented in Figs. 6 and 7 for SNR values of $3.7 \mathrm{~dB}$ and $10.5 \mathrm{~dB}$, respectively. These correspond to the SNR requirement of the modulation schemes to achieve an uncoded BER target of $10^{-3}$, sufficient to enable FEC, in the linear channel with AWGN, where the gain of $6.1 \mathrm{~dB}$ in the linear region of the SSPA from Fig. 1 is considered. Due to the minimal constellation warping in QPSK, the receivers show similar performance for given number of iterations and transmission scheme. Without IMI cancellation, the improved receiver shows a lower TD for higher order constellations, e.g., up to $0.2 \mathrm{~dB}$ for 16 -QAM with TDMA with $20 \%$ roll-off. When 
TD $v s$. OBO of TDMA, SC-FDMA and OFDMA in AWGN with SSPA in RTN link, $E / N_{0}=3.7 \mathrm{~dB}$

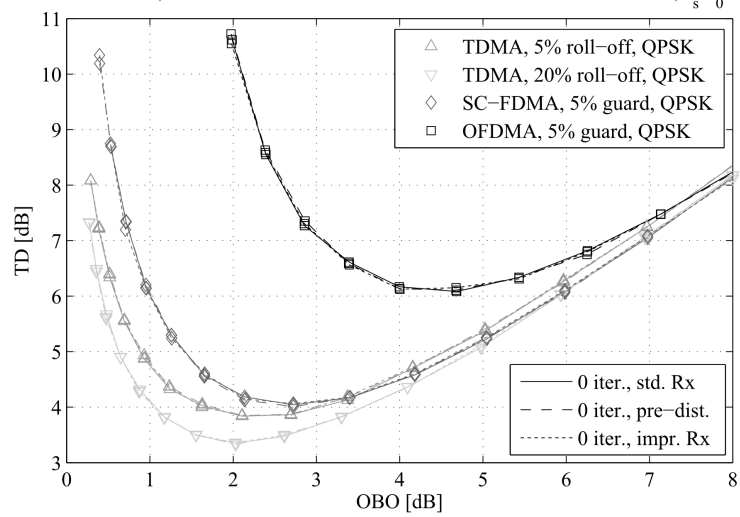

(a) No iterations.

TD vs. OBO of TDMA, SC-FDMA and OFDMA in AWGN with SSPA in RTN link, $E / E_{0}=3.7 \mathrm{~dB}$

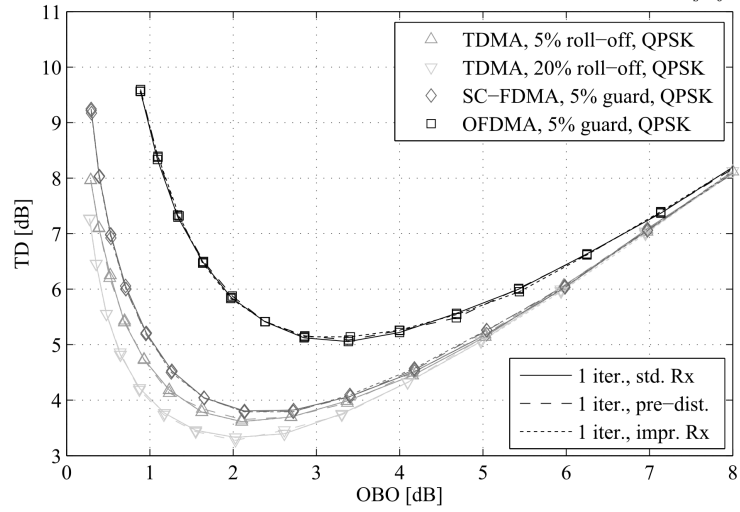

(b) One iteration.

TD $v s$. OBO of TDMA, SC-FDMA and OFDMA in AWGN with SSPA in RTN link, $E_{\mathrm{s}} / N_{0}=3.7 \mathrm{~dB}$

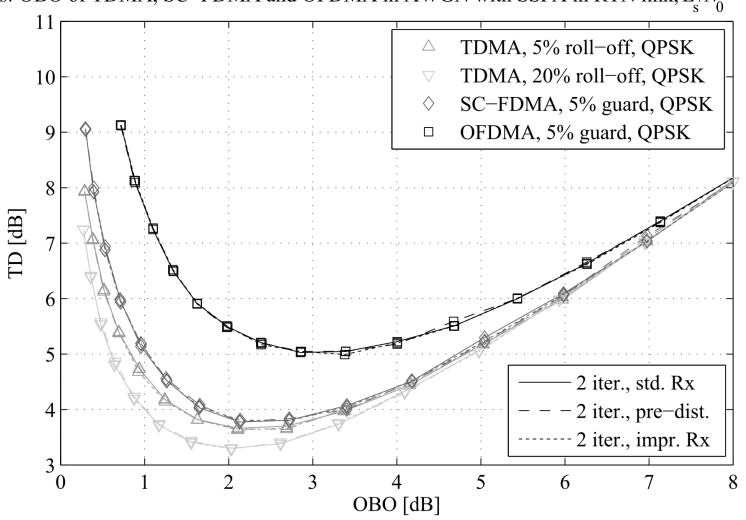

(c) Two iterations.

Fig. 6: TD vs. OBO of digital transmission schemes for QPSK in the satellite return link.

iterative IMI cancellation is employed, the improved receiver consistently outperforms the standard receiver with or without pre-distortion for all OBO values. This is due to the fact that data pre-distortion increases the PAPR of the waveform which increases the distortion noise [4-6]. TDMA with $20 \%$ roll-off shows the lowest TD in the non-linear channel followed by SC-FDMA and OFDMA. In addition, SC-FDMA is able to outperform TDMA with $5 \%$ roll-off for higher constellation

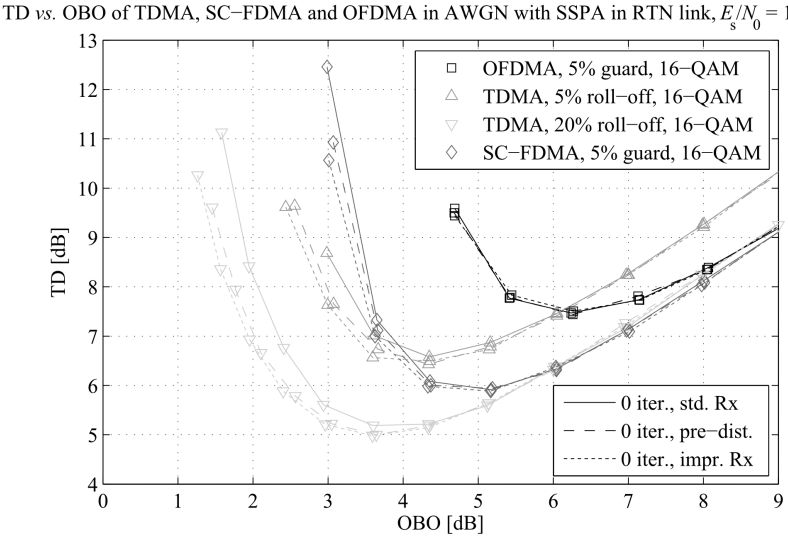

(a) No iterations.

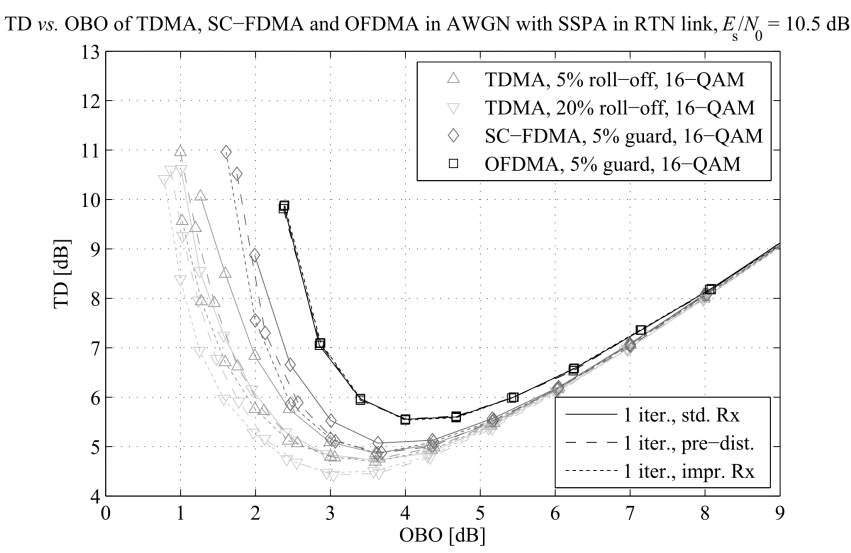

(b) One iteration.

TD $v s$. OBO of TDMA, SC-FDMA and OFDMA in AWGN with SSPA in RTN link, $E_{\mathrm{s}} / N_{0}=10.5 \mathrm{~dB}$

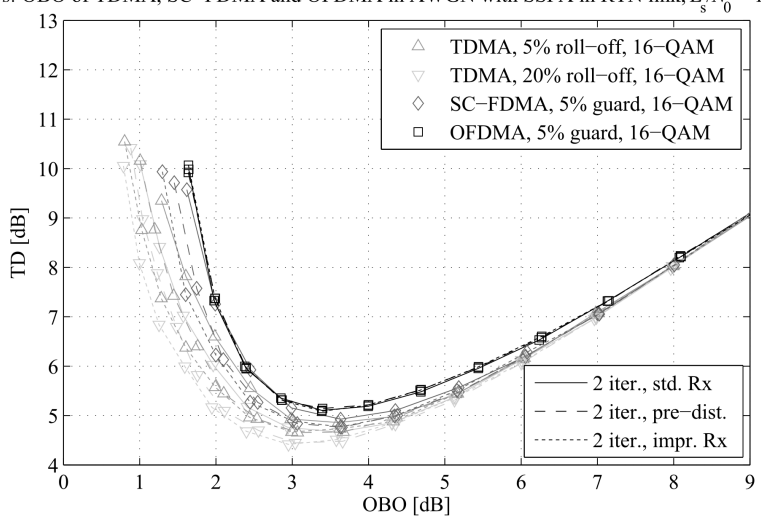

(c) Two iterations.

Fig. 7: TD vs. OBO of digital transmission schemes for 16-QAM in the satellite return link.

orders due to the residual non-linear distortion noise for very low roll-off factors, resulting from the truncation of the SRRCF impulse response. The improved receiver shifts the minimum TD values to lower OBO values as shown in TABLE I for 16-QAM. The optimum OBO is reduced from $3.6 \mathrm{~dB}$ to $2.95 \mathrm{~dB}$ in TDMA with $20 \%$ roll-off, from $4.35 \mathrm{~dB}$ to $3 \mathrm{~dB}$ in TDMA with $5 \%$ roll-off, from $5.17 \mathrm{~dB}$ to $3.63 \mathrm{~dB}$ in SC-FDMA, and from $6.25 \mathrm{~dB}$ to $3.39 \mathrm{~dB}$ in OFDMA. 


\begin{tabular}{|l|l|l|l|l|l|l|}
\hline Number of iterations & \multicolumn{2}{|c|}{ No iterations } & \multicolumn{2}{c|}{ One iteration } & \multicolumn{2}{c|}{ Two iterations } \\
\hline Receiver & std. Rx & impr. Rx & std. Rx & impr. Rx & std. Rx & impr. Rx \\
\hline TDMA, 20\% roll-off & $5.19 @ 3.6$ & $4.98 @ 3.6$ & $4.72 @ 3.6$ & $4.47 @ 2.95$ & $4.67 @ 3.6$ & $4.42 @ 2.95$ \\
\hline TDMA, 5\% roll-off & $6.58 @ 4.35$ & $6.49 @ 4.35$ & $4.87 @ 3.6$ & $4.69 @ 3.6$ & $4.86 @ 3.6$ & $4.67 @ 3$ \\
\hline SC-FDMA, 5\% guard & $5.91 @ 5.17$ & $5.89 @ 5.17$ & $5.07 @ 3.64$ & $4.86 @ 3.62$ & $4.93 @ 3.63$ & $4.77 @ 3.63$ \\
\hline OFDMA, 5\% guard & $7.5 @ 6.25$ & $7.5 @ 6.25$ & $5.54 @ 4$ & $5.54 @ 4$ & $5.01 @ 3.39$ & $5.01 @ 3.39$ \\
\hline
\end{tabular}

TABLE I: Minimum TD [dB] @ optimum OBO [dB] of TDMA, SC-FDMA and OFDMA waveforms with 16-QAM in the satellite return link.

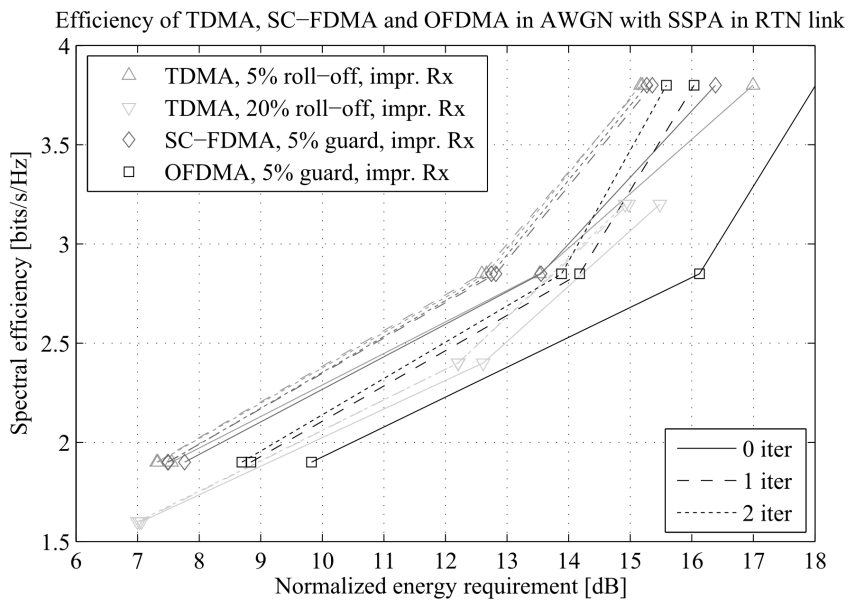

Fig. 8: Spectral efficiency vs. normalized energy requirement of TDMA, SC-FDMA and OFDMA waveforms in the satellite return link.

In this study, the normalized energy requirement of the waveforms is defined as the summation of the TD values and the reference SNRs. Taking into account the roll-off factors and the guard bands, the spectral efficiency is presented as a function of the normalized energy requirement in Fig. 8 for the improved receiver. Here, also 8-PSK with an SNR of 8.9 $\mathrm{dB}$ is included. SC-FDMA and TDMA with $5 \%$ roll-off are shown to be the most spectrally efficient transmission scheme in the tested scenarios with the improved IMI cancelling receiver, while it allows OFDMA to close the gap for higher order modulation. In summary, the improved receiver with 2 iterations of IMI cancellation shows the following gains relative to the standard receiver without IMI cancellation: $0.1-0.8 \mathrm{~dB}$ for TDMA with $20 \%$ roll-off, $0.2-1.9 \mathrm{~dB}$ for TDMA with $5 \%$ roll-off, $0.3-1.1 \mathrm{~dB}$ for SC-FDMA, and $1.1-2.5 \mathrm{~dB}$ for OFDMA.

\section{CONCLUSION}

In this paper, an iterative receiver has been presented to cancel the IMI that originates from the non-linear distortion in the satellite return link channel. The receiver has been tested with TDMA, SC-FDMA and OFDMA transmission formats, and it has demonstrated significant gains in the energy efficiency. It has been shown that two, or even one, iterations are sufficient to present the majority of the gain of IMI cancellation, resulting in a low complexity overhead.
The improved receiver enables the application of lower OBO settings of the SSPA at the user terminal, and it is suitable for integration in low-cost devices. In the considered modulation setup, total degradation gains of up to $2.5 \mathrm{~dB}$ are expected for OFDMA, $1.1 \mathrm{~dB}$ for SC-FDMA, $0.8 \mathrm{~dB}$ for TDMA with $20 \%$ roll-off, and $1.9 \mathrm{~dB}$ for TDMA with $5 \%$ roll-off. The receiver is particularly suitable for application with higher order modulation formats which are more vulnerable to nonlinear distortion, where even higher energy efficiency gains are expected.

\section{ACKNOWLEDGEMENT}

This work has been supported by the BATS research project funded by the EU-FP7 under contract n317533.

\section{REFERENCES}

[1] Broadband Access via Integrated Terrestrial and Satellite Systems (BATS), "ICT-2011.1.1 BATS D4.1: Satellite Network Mission Requirements," European Project, Tech. Rep., 2012.

[2] Second Generation DVB Interactive Satellite System (DVB-RCS2); Part 2: Lower Layers for Satellite Standard, Digital Video Broadcasting (DVB) Std. ETSI EN 301 545-2, Jan. 2012.

[3] V. Dalakas, P. Mathiopoulos, F. Do Cecca, and G. Gallinaro, "A Comparative Study Between SC-FDMA and OFDMA Schemes for Satellite Uplinks," IEEE Transactions on Broadcasting, vol. 58, no. 3, pp. 370-378, Sep. 2012.

[4] E. Casini, R. De Gaudenzi, and A. Ginesi, "DVB-S2 Modem Algorithms Design and Performance Over Typical Satellite Channels," International Journal of Satellite Communications and Networking, vol. 22, pp. 281318, Jun. 2004

[5] G. Karam and H. Sari, "Analysis of Predistortion, Equalization, and ISI Cancellation Techniques in Digital Radio Systems with Nonlinear Transmit Amplifiers," IEEE Transactions on Communications, vol. 37, no. 12 , pp. $1245-1253$, Dec. 1989.

[6] — "A Data Pre-distortion Technique with Memory for QAM Radio Systems," IEEE Transactions on Communications, vol. 39, no. 2, pp. 336-344, Aug. 1991.

[7] N. Kelly, M. Allegue-Martinez, P.-D. Arapoglou, and A. Zhu, "Bandwidth-constrained Digital Pre-distortion Technique for Multicarrier Satellite Communications," International Journal of Satellite Communications and Networking, Apr. 2015.

[8] H. Chen and A. M. Haimovich, "Iterative Estimation and Cancellation of Clipping Noise for OFDM Signals," IEEE Communications Letters, vol. 7, no. 7, pp. 305-307, Jul. 2003.

[9] J. Gazda, P. Drotar, M. Deumal, D. Kocur, and P. Galajda, "Simple Iterative Cancellation of Nonlinear Distortion in LDFMA Systems," in Proc. of 14th International OFDM Workshop 2009, Hamburg, Germany, Apr. 2009, pp. 133-137.

[10] J. Bussgang, "Cross Correlation Function of Amplitude-Distorted Gaussian Signals,' Research Laboratory for Electronics, Massachusetts Institute of Technology, Cambridge, MA, Technical Report 216, Mar. 1952.

[11] Technical Specification Group Radio Access Network; Evolved Universal Terrestrial Radio Access (EUTRA); User Equipment (UE) Radio Transmission and Reception, 3rd Generation Partnership Project Std. 3GPP TS 36.101, 2011

[12] R. Djardin, M. Colas, and G. Gelle, "Comparison of Iterative Receivers Mitigating the Clipping Noise of OFDM Based System," in Proc. of European Wireless Conference 2007, Paris, France, Apr. 2007. 